\title{
1 Consistent assimilation of multiple data streams in a \\ 2 carbon cycle data assimilation system
}

3

4 Natasha MacBean ${ }^{1}$, Philippe Peylin ${ }^{1}$, Frédéric Chevallier ${ }^{1}$, Marko Scholze ${ }^{2}$,

5 Gregor Schürmann ${ }^{3}$

6 [1] \{Laboratoire des Sciences du Climat et de 1'Environnement, LSCE/IPSL, CEA-CNRS-

7 UVSQ, Université Paris-Saclay, F-91191 Gif-sur-Yvette, France\}

8 [2]\{Department of Physical Geography and Ecosystem Science, Lund University, Lund,

9 Sweden

10 [3] Max Planck Institute for Biogeochemistry, Jena, Germany\}

11 Correspondence to: N. MacBean (nlmacbean@gmail.com)

12

13 Supplementary material
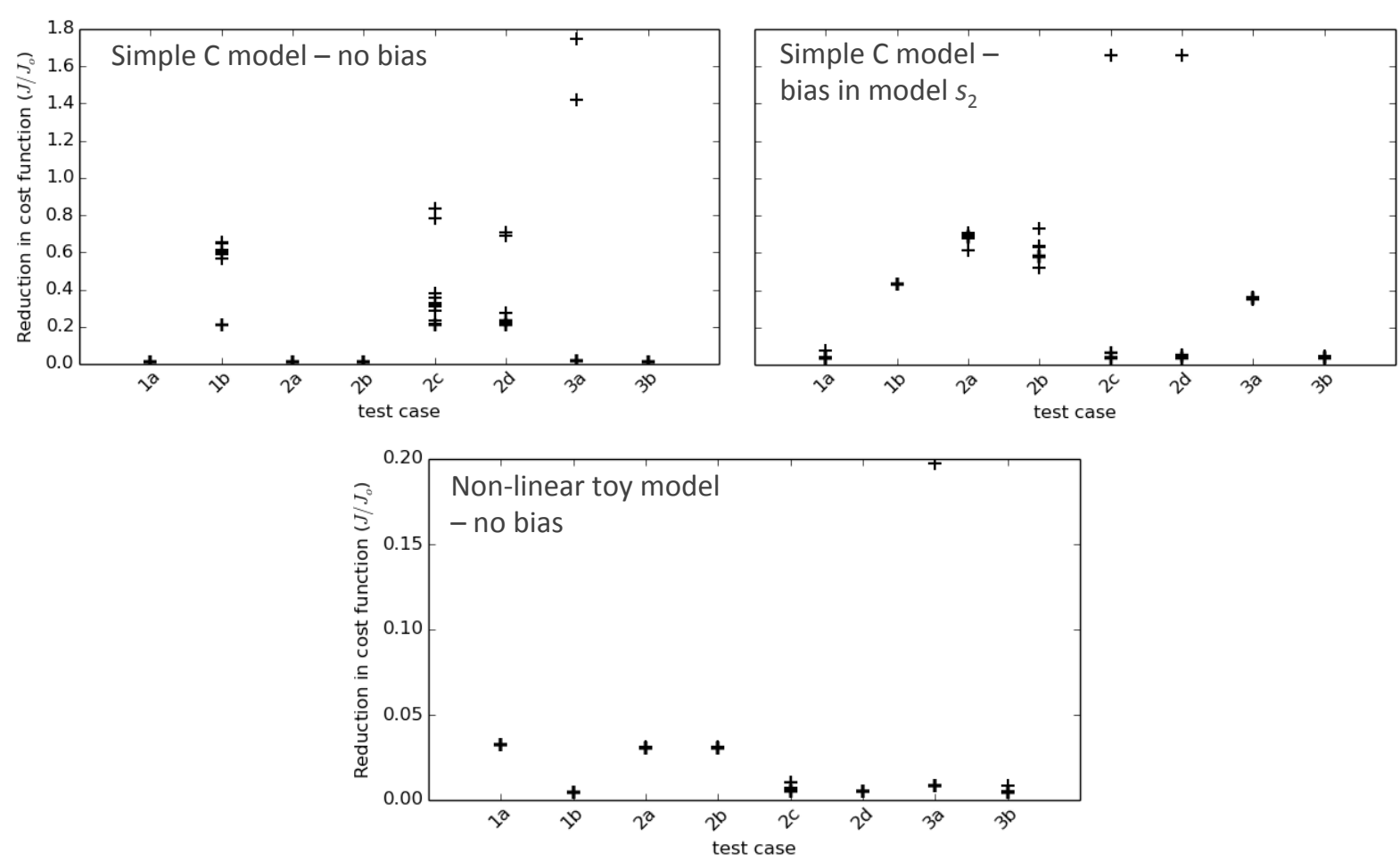

14

Figure S1: Reduction in the cost function $\left(J / J_{o}\right)$ for each model and each test for all 20

16 assimilations with different random "first guess" points in the parameter space (i.e. each cross 
1 represents the 20 random "first guess" tests). Top panel - simple C model without bias (left)

2 and with bias added to the simulated $s_{2}$ variable (right). Bottom panel - non-linear toy model

3 with no added bias. Note that the majority of the random "first guess" assimilations achieve

4 the same reduction in the cost function even though the final value is different for each test,

5 which is to be expected as each test (for each model) has a different cost function. 